\title{
Insights into activation and dimerization of soluble guanylate cyclase
}

\author{
Christiane Rothkegel*1,2, Peter M Schmidt ${ }^{3}$, Derek-John Atkins ${ }^{1,4}$, \\ Friederike Stoll ${ }^{1}$, Henning Schröder ${ }^{2}$, Harald HHW Schmidt ${ }^{3}$ and Johannes- \\ Peter Stasch ${ }^{1}$
}

\begin{abstract}
Address: ${ }^{1}$ Pharma Research Center, Bayer HealthCare, Aprather Weg 18a, 42096 Wuppertal, Germany, ${ }^{2}$ Martin-Luther-University, School of Pharmacy, Wolfgang-Langenbeck-Strasse 4, 06120 Halle, Germany, ${ }^{3}$ Department of Pharmacology, Monash University, Melbourne, Clayton, VIC 3800, Australia and ${ }^{4}$ Helios Klinkum, Institute for Pathology, Heusnerstrasse 40, D-42283 Wuppertal, Germany

Email: Johannes-Peter Stasch - johannes-peter.stasch@bayerhealthcare.com

* Corresponding author
\end{abstract}

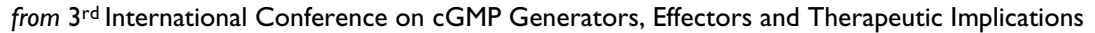
Dresden, Germany. 15-17 June 2007

Published: 25 July 2007

BMC Pharmacology 2007, 7(SuppI I):S36 doi:I0.I I86/I47I-22I0-7-SI-S36

This abstract is available from: http://www.biomedcentral.com/I47I-22/0/7/SI/S36

(c) 2007 Rothkegel et al; licensee BioMed Central Ltd.

The intracellular nitric oxide receptor, soluble guanylate cyclase (sGC), is an obligate heterodimer of an $\alpha$ - and a smaller, heme-containing $\beta$-subunit. The prosthetic heme group is essential for the enzyme's NO-sensing capability and heme-free sGC has been shown to be unesponsive to NO. The generation of a cGMP reporter cell line together with the NO-independent but heme-dependent sGC stimulator BAY 41-2272, the NO- and heme-independent sGC activator BAY 58-2667, NO, and ODQ enabled us for the first time to distinguish between both sGC states in intact cells. Investigation of the activation profile of different sGC mutants by transient transfection into this CGMP reporter cell led to the identification of the heme binding motif $\operatorname{Tyr}_{135}-\mathrm{x}-\operatorname{Ser}_{137}-\mathrm{x}-\operatorname{Arg}_{139}$ in addition to the axial heme-ligand $\mathrm{His}_{105}$. Mutational analysis of the $\beta$ hemebinding site identified the amino acids $\beta A s_{44}, \beta A s p_{45}$ and $\beta \mathrm{Phe}_{74}$ as being crucially important for the heme-induced sGC activation.

Heterodimerization of the $\alpha / \beta$-subunits has been proposed to be mediated mainly by the central region of sGC based on the homology of amino acids to the sequence mediating particulate guanylate cyclase homodimerization and, in addition, coprecipation studies with sGC. However, the methodical limitations of these techniques foiled the accurate identification of segments contributing to the sGC heterodimerization. To overcome these obstacles we applied the recently developed method of bimolecular fluorescence complementation (BiFC). BiFC is based on the ability of two nonfluorescent fragments of YFP to form the functional fluorophor upon interaction of the fused $\alpha 1$ - and $\beta 1$-sGC subunits. This technique enabled us to map stretches involved in the process of heterodimerization in a non-invasive manner directly in the intracellular environment. A screen of the heterodimeric profile of small deletion mutants revealed that segments spanning amino acids $\alpha_{1} 363-372, \alpha_{1} 403-422, \alpha_{1} 440-$ 459 and $\beta_{1} 212-222, \beta_{1} 304-333, \beta_{1} 344-363, \beta_{1} 381-400$ within the predicted dimerization region are involved in the process of heterodimerization. In conclusion it is evident that the dimerization region of the sGC $\alpha$ and $\beta$ subunit consists of discontinuous binding modules as already postulated for $\beta_{1}$-sGC based on coprecipitation studies. Moreover, the BiFC study supports the hypothesis that the interaction of $\alpha_{1} / \beta_{1}$-sGC is at least partially mediated by an amphipathic $\alpha$-helical structure. In summary, our studies demonstrate that the CGMP readout cell line in combination with the modulators of sGC activity and the recently developed method of BiFC represent powerful tools to elucidate the process of sGC dimerization and activation. 